\title{
THE SCALED BOUNDARY FINITE ELEMENT METHOD FOR THE EFFICIENT MODELLING OF LINEAR ELASTIC FRACTURE
}

\author{
ADRIAN W. EGGER* ${ }^{*}$ SAVVAS P. TRIANTAFYLLOU ${ }^{\dagger}$ AND ELENI N. CHATZI* \\ ${ }^{*}$ Swiss Federal Institute of Technology Zurich (ETHZ) \\ Zurich, Switzerland \\ e-mail: egger@ibk.baug.ethz.ch and chatzi@ibk.baug.ethz.ch \\ ${ }^{\dagger}$ University of Nottingham \\ Nottingham, UK \\ e-mail: savvas.triantafyllou@ @ottingham.ac.uk
}

Key words: Scaled Boundary Finite Element Method (SBFEM), eXtended Finite Element Method (XFEM), Linear Elastic Fracture Mechanics (LEFM), Stress Intensity Factors (SIFs), Multiscale

\begin{abstract}
In this work, a study of computational and implementational efficiency is presented, on the treatment of Linear Elastic Fracture Mechanics (LEFM) problems. To this end, the Scaled Boundary Finite Element Method (SBFEM), is compared against the popular eXtended Finite Element Method (XFEM) and the standard FEM approach for efficient calculation of Stress Intensity Factors (SIFs). The aim is to examine SBFEM's potential for inclusion within a multiscale fracture mechanics framework. The above features will be exploited to solve a series of benchmarks in LEFM comparing XFEM, SBFEM and commercial FEM software to analytical solutions. The extent to which the SBFEM lends itself for inclusion within a multiscale framework will further be assessed.
\end{abstract}

\section{INTRODUCTION}

The possibility of modelling damage related phenomena, such as crack initiation, crack propagation and de-lamination by finite element method (FEM) has been demonstrated in [1]. Additionally, a significant amount of research has been conducted specifically on the topic of singular elements [2] in order to properly capture the stress singularities about crack tips. However, certain issues such as extensive mesh refinement, remeshing and mesh dependent projection errors have been encountered, rendering this method undesirable in terms of implementational complexity and accuracy, as well as computationally prohibitive for larger systems.

The aforementioned issues inherent to FEM have given rise to the eXtended Finite Element
Method (XFEM) [3]. Many shortcomings, such as the excessive mesh refinement and mesh dependent projection errors of the standard FEM formulations were alleviated. The principle idea lies in decoupling the discontinuities, both weak (material interfaces) and strong (cracks), from the mesh. This is achieved via adoption of the level set method (LSM) [4] for tracking discontinuities in conjunction with the seeding of jump and crack tip enrichments to the displacement solution, thus extending the standard FEM procedure. Leaving intact the core concepts and steps of FEM is in large responsible for its success and inclusion in most major commercial FEM software packages.

Though XFEM has garnered significant academic interest and resolved major issues associated with FEM based damage simulation in the process, it is still an evolving field of research. 
A select few open topics include challenges extending the method to 3D applications with respect to the LSM [5], the rapid increase in condition number of the stiffness matrix [6] and the need for a priori knowledge of the type of crack tip singularity before solution [7].

Another numerical method, also based on FEM, which lends itself naturally to the task of modelling damage related phenomena without the need for an extension, is the scaled boundary finite element method (SBFEM).

The original publication of Dasgupta [8], coining the term "Cloning Algorithm", marks SBFEM's beginnings for modelling wave propagation in unbounded soils, i.e., for soilstructure interaction and earthquake engineering. Wolf built upon this work naming it the "infinitesimal finite element method" [9]. Through a series of advances ([10], [11]), he and his collaborators arrived at the formulation based on a derivation using the minimum weighted residual method named SBFEM. A collaboration with Deeks [12] led to the derivation of the SBFEM equations by virtual work formulation.

Originally intended for unbounded domains it was soon found that SBFEM is equally effective for bounded domains [11] as well as damage related phenomena. By further utilizing the Schur decomposition in conjunction with a matrix exponential during the solution process [13], the numerical issues with repeated eigenvalues associated with the standard eigenvalue decomposition are eliminated. Further, the power of the crack tip singularity is accurately accounted for, which allows quantifying the stress intensity factors (SIFs) at material interfaces with greater precision. Based on these findings, crack propagation algorithms have been proposed ([14], [15], [16]).

Additional topics of note include the treatment of dynamics based on a continued fraction solution [17], a Frobenius series expansion [18] and the spectral element method [19]. Recently, SBFEM has been extended to material non-linearity [20] as well as material and geometric non-linearity [21]. Further, a non-linear heat transfer problem was solved using the ho- motopy analysis method (HAM) [22]. Also, hybrid methods such as coupling with meshless methods [23], boundary element method (BEM) [24] and the finite element method (FEM) [25] have been developed. Furthermore, SBFEM has been used as crack tip enrichment in XFEM [26]. In addition, isogeometric analysis has successfully been fused with the SBFEM principles [27]. Finally, a novel variation of SBFEM with diagonalized and thus decoupled coefficient matrices has been developed [28], in which each node can be treated independently.

As SBFEM lends itself naturally to the solution of damage related phenomena it remains to be determined how it compares to XFEM in this regard. Unfortunately, very little literature exists on this topic. The authors are only aware of two publications that in passing touch on this subject matter. One compares a polygon based SBFEM to XFEM directly [15], the other compares XFEM with SBFEM as tip enrichment to standard XFEM [26]. Unfortunately, based on these publications no comprehensive conclusions can be made, which affirms the need for a more thorough investigation.

As a result, this paper is organized as follows: Section 2 presents the underlying theory of SBFEM with focus on the SIFs. Section 3 contains numerical examples, wherein the performance of SBFEM is compared to analytical and references solutions as well as published benchmark problems. Finally, the findings are summarized in section 4 .

\section{THEORY}

The strong form of the governing equations in 2D elastostatics is given as follows:

$$
\begin{aligned}
& \text { equilibrium: } \quad \boldsymbol{L} \boldsymbol{\sigma}+\boldsymbol{b}=\mathbf{0} \quad \text { in } \Omega \\
& \text { constitutive: } \quad \sigma=D \epsilon \quad \text { in } \Omega \\
& \text { compatability: } \quad \boldsymbol{\epsilon}=\boldsymbol{L}^{T} \boldsymbol{u} \quad \text { in } \Omega \\
& \text { boundary conditions: } \boldsymbol{u}=\hat{\boldsymbol{u}} \quad \text { on } \Gamma \\
& \boldsymbol{\sigma} \cdot \boldsymbol{n}=\hat{\boldsymbol{t}} \quad \text { on } \Gamma
\end{aligned}
$$

First, the elastostatic equilibrium equations of the discrete problem are formulated by means of the Principle of Virtual Work resulting in the scaled boundary finite element equation in elas- 
tostatics. Next, the solution procedure, which leads to the formulation of the stiffness matrix will be presented. Then, as part of the postprocessing, the computation of the stress intensity factors (SIFs) will be shown.

\subsection{Summary of SBFEM}

SBFEM's unique properties originate from the introduction of a scaling center $O$, which, in all but a few special cases, must be directly visible from every point on the domain boundary. In doing so, the Cartesian coordinate system is transformed into one resembling polar coordinates. Thus, a radial coordinate $\xi$ and a tangential coordinate $\eta$ are introduced. Based on the premise that an analytical solution can be found in the radial direction and therefore only the tangential direction must be discretized in the finite element sense, $\xi$ runs from $0 \leq \xi \leq 1$ for bounded domains, $1 \leq \xi \leq \infty$ for unbounded domains and $\xi_{\text {interior }} \leq \xi \leq \xi_{\text {exterior }}$ for bounded domains consisting of similar boundaries, whereas $\eta$ spans $-1 \leq \eta \leq 1$ for each element.
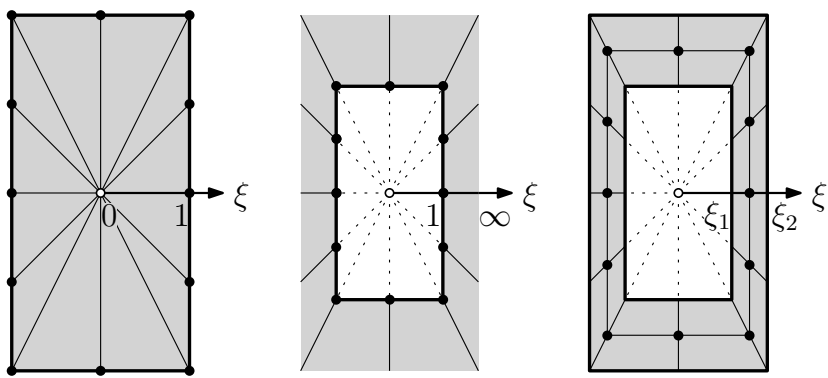

- scaling center

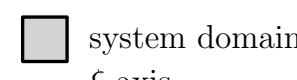

- boundary node

$\xi$-axis

Figure 2: Possible domain types using SBFEM

By consequence, it is only necessary to discretize the boundary, which reduces the dimension of the problem by one. As a result, each element includes additional information pertaining to the portion of the domain spanned by itself and the scaling center (Fig. 2).

\subsubsection{Derivation based on the virtual work approach}

The change of coordinates from Cartesian $(x, y)$ to scaled boundary $(\xi, \eta)$ is termed the scaled boundary transformation of geometry. It states that any point in the domain is therefore equivalently mapped as:

$$
\begin{aligned}
& x(\xi, \eta)=x_{O}+\xi x(\eta)=x_{O}+\xi[\boldsymbol{N}(n)]\{\boldsymbol{x}\} \\
& y(\xi, \eta)=y_{O}+\xi y(\eta)=y_{O}+\xi[\boldsymbol{N}(n)]\{\boldsymbol{y}\}
\end{aligned}
$$

where $[\boldsymbol{N}(\eta)]$ corresponds to the finite element interpolation functions $\left[N_{1}(\eta), N_{2}(\eta), \ldots, N_{n}(\eta)\right],\{\boldsymbol{x}\}$ resp. $\{\boldsymbol{y}\}$ represents the nodal coordinates $\left[x_{1}, x_{2}, \ldots, x_{n}\right]^{T}$ and $n$ is the number of nodes per element on the boundary. The derivatives relating the Cartesian to the scaled boundary coordinate system are linked by the Jacobian $\boldsymbol{J}$, its inverse $\boldsymbol{J}^{-1}$ and determinant $|\boldsymbol{J}|$ all at the boundary as expressed below:

$$
\begin{aligned}
& {\left[\begin{array}{l}
\bullet, \xi \\
\bullet, \eta
\end{array}\right]=\quad\left[\begin{array}{ll}
x, \xi & y_{,} \\
x, \eta & y_{\eta}
\end{array}\right]\left[\begin{array}{l}
\bullet \\
,_{x} \\
\bullet, y
\end{array}\right]=\boldsymbol{J} \quad\left[\begin{array}{l}
\bullet \\
\bullet \\
\bullet, y
\end{array}\right]} \\
& {\left[\begin{array}{l}
\bullet, x \\
\bullet
\end{array}\right]=\frac{1}{|\boldsymbol{J}|}\left[\begin{array}{rr}
y_{,} & -y, \xi \\
-x, \eta & x, \xi
\end{array}\right]\left[\begin{array}{l}
\bullet, \xi \\
\bullet, \eta
\end{array}\right]=\boldsymbol{J}^{-1}\left[\begin{array}{l}
\bullet, \xi \\
\bullet, \eta
\end{array}\right]}
\end{aligned}
$$

where the notation $\bullet \boldsymbol{}_{x}$ is introduced as the derivative with respect to $\mathrm{x}$.

Consequently, a differential unit of volume $d V$ may be formulated in scaled boundary coordinates as:

$$
d V=|\boldsymbol{J}| \xi d \xi d \eta
$$

The derivatives may further be used to construct the linear differential operator $\boldsymbol{L}$ in scaled boundary coordinates. In a first step, it is split. Then the partial derivatives are substituted, which results in the following formulation:

$$
[\boldsymbol{L}]=\left[\boldsymbol{b}^{\mathbf{1}}(\eta)\right] \cdot \boldsymbol{\bullet}, \xi+\frac{1}{\xi}\left[\boldsymbol{b}^{\mathbf{2}}(\eta)\right] \cdot \bullet, \eta
$$

where $\left[\boldsymbol{b}^{\mathbf{1}}(\eta)\right]$ and $\left[\boldsymbol{b}^{\mathbf{2}}(\eta)\right]$ represent:

$$
\begin{array}{r}
{\left[\boldsymbol{b}^{\mathbf{1}}(\eta)\right]=\frac{1}{|\boldsymbol{J}|}\left[\begin{array}{rr}
y_{, \eta} & 0 \\
0 & -x, \eta \\
-x, \eta & y, \eta
\end{array}\right]} \\
{\left[\boldsymbol{b}^{\mathbf{2}}(\eta)\right]=\frac{1}{|\boldsymbol{J}|}\left[\begin{array}{rr}
-y, \xi & 0 \\
0 & x, \xi \\
x, \xi & -y, \xi
\end{array}\right]}
\end{array}
$$




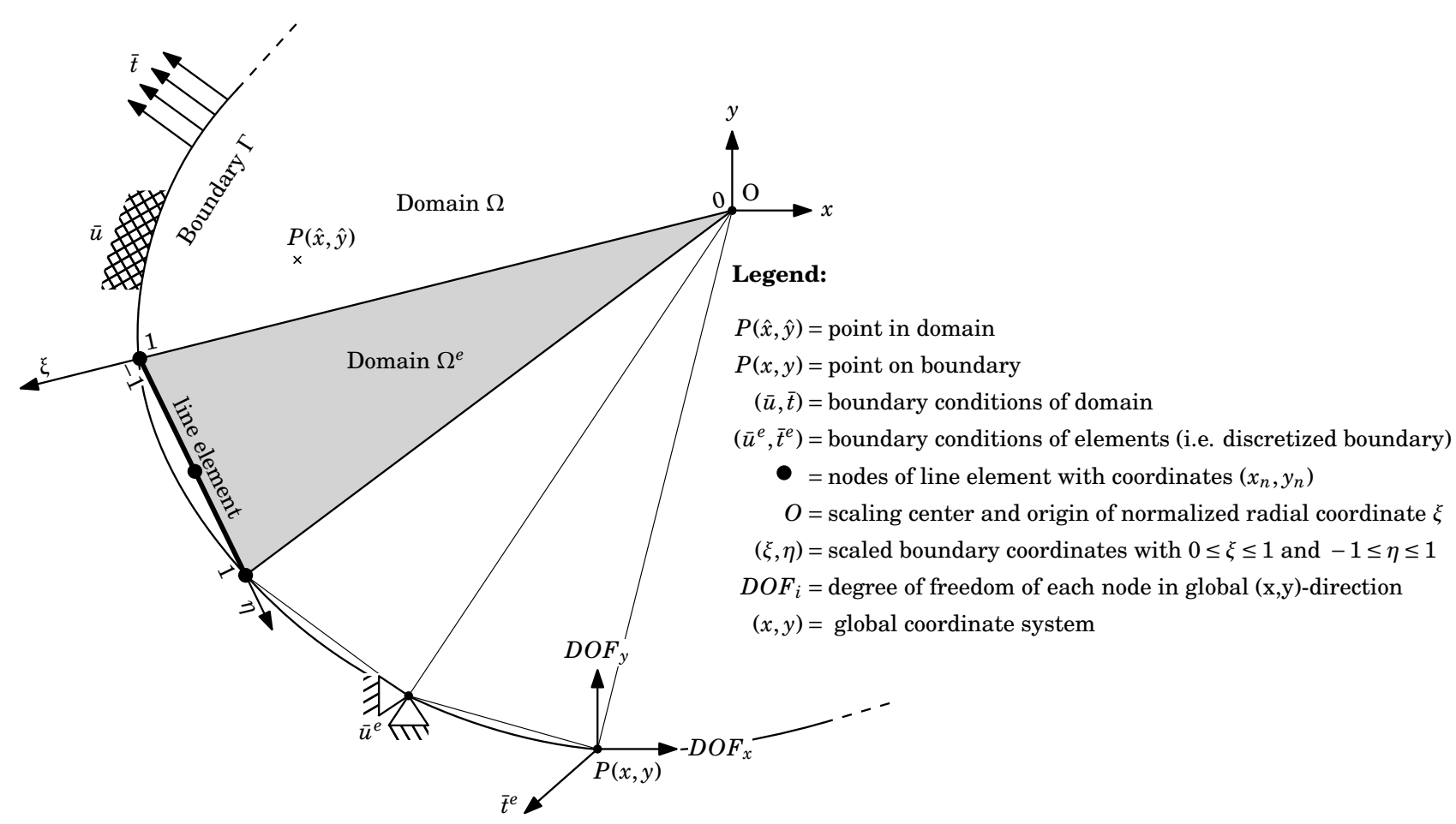

Figure 1: Introduction of SBFEM specific discretization of the domain

The displacements on the boundary are interpolated in an isoparametric fashion. Analogous to the geometry mapping the displacements $\{\boldsymbol{u}(\xi, \eta)\}$ are comprised of an analytical part in radial direction $\{\boldsymbol{u}(\xi)\}$ and an interpolatory part relying on interpolation functions $\left[\boldsymbol{N}^{\boldsymbol{u}}(\eta)\right]$ in tangential direction:

$$
\{\boldsymbol{u}(\xi, \eta)\}=\left[\boldsymbol{N}^{\boldsymbol{u}}(\eta)\right]\{\boldsymbol{u}(\xi)\}
$$

where $\left[\boldsymbol{N}^{\boldsymbol{u}}(\eta)\right]$ represents the shape functions $[\boldsymbol{N}(\eta)]$ applied to each degree of freedom independently by applying the identity matrix $\boldsymbol{I}$ as follows:

$$
\left[\boldsymbol{N}^{\boldsymbol{u}}(\eta)\right]=\left[N^{1}(\eta) \boldsymbol{I}_{\boldsymbol{n}}, N^{2}(\eta) \boldsymbol{I}_{\boldsymbol{n}}, \ldots, N^{n}(\eta) \boldsymbol{I}_{\boldsymbol{n}}\right]
$$

Hence, by substituting into eqn. $1 \mathrm{c}$ an expression for the strains may be formulated:

$$
\{\boldsymbol{\epsilon}(\xi, \eta)\}=\left[\boldsymbol{B}^{\mathbf{1}}(\eta)\right]\{\boldsymbol{u}(\xi)\}, \xi+\frac{1}{\xi}\left[\boldsymbol{B}^{\mathbf{2}}(\eta)\right]\{\boldsymbol{u}(\xi)\}
$$

where

$$
\begin{aligned}
{\left[\boldsymbol{B}^{\mathbf{1}}(\eta)\right] } & =\left[\boldsymbol{b}^{\mathbf{1}}(\eta)\right]\left[\boldsymbol{N}^{\boldsymbol{u}}(\eta)\right] \\
{\left[\boldsymbol{B}^{\mathbf{2}}(\eta)\right] } & =\left[\boldsymbol{b}^{\mathbf{2}}(\eta)\right]\left[\boldsymbol{N}^{\boldsymbol{u}}(\eta)\right]_{, \eta}
\end{aligned}
$$

And as a consequence the stresses follow as:

$$
\{\boldsymbol{\sigma}(\xi, \eta)\}=[\boldsymbol{D}]\left(\left[\boldsymbol{B}^{\mathbf{1}}(\eta)\right]\{\boldsymbol{u}(\xi)\}_{, \xi}+\frac{1}{\xi}\left[\boldsymbol{B}^{\mathbf{2}}(\eta)\right]\{\boldsymbol{u}(\xi)\}\right)
$$

Choosing to proceed by means of the principle of virtual work, the corresponding virtual displacements and strains are expressed as:

$$
\begin{aligned}
\{\delta \boldsymbol{u}(\xi, \eta)\} & =[\boldsymbol{N}(\boldsymbol{\eta})]\{\delta \boldsymbol{u}(\xi)\} \\
\{\delta \boldsymbol{\epsilon}(\xi, \eta)\} & =[\boldsymbol{L}]\{\delta \boldsymbol{u}(\xi, \eta)\}
\end{aligned}
$$

As such, the virtual work statement equating internal work to external work in scaled boundary coordinates becomes:

$$
\int_{V}\{\delta \boldsymbol{\epsilon}(\xi, \eta)\}^{T}\{\boldsymbol{\sigma}(\xi, \eta)\} d V-\int_{\partial \Omega}\{\delta \boldsymbol{u}(\eta)\}^{T}\{\boldsymbol{t}(\eta)\}=0
$$

Proceeding analogously as in the case for standard FEM, two equations arise:

$$
\begin{array}{r}
\{\boldsymbol{P}\}=\left[\boldsymbol{E}^{\mathbf{0}}\right]\left\{\boldsymbol{u}_{\boldsymbol{h}}\right\}_{, \xi}+\left[\boldsymbol{E}^{\mathbf{1}}\right]^{T}\left\{\boldsymbol{u}_{\boldsymbol{h}}\right\} \\
{\left[\boldsymbol{E}^{\mathbf{0}}\right] \xi^{2}\{\boldsymbol{u}(\xi)\}_{, \xi \xi}+\left[\left[\boldsymbol{E}^{\mathbf{0}}\right]+\left[\boldsymbol{E}^{\mathbf{1}}\right]^{T}-\left[\boldsymbol{E}^{\mathbf{1}}\right]\right] \xi\{\boldsymbol{u}(\xi)\}_{, \xi}} \\
-\left[\boldsymbol{E}^{\mathbf{2}}\right]\{\boldsymbol{u}(\xi)\}=\{\mathbf{0}\}
\end{array}
$$

and the following substitutions are introduced for brevity, which bare striking similarity to stiffness matrices in FEM:

$$
\begin{aligned}
& {\left[E^{0}\right]=\int_{\partial \Omega}\left[\boldsymbol{B}^{1}(\eta)\right]^{T}[\boldsymbol{D}]\left[\boldsymbol{B}^{1}(\eta)\right]|\boldsymbol{J}| d \eta} \\
& {\left[E^{1}\right]=\int_{\partial \Omega}\left[\boldsymbol{B}^{1}(\eta)\right]^{T}[\boldsymbol{D}]\left[\boldsymbol{B}^{2}(\eta)\right]|\boldsymbol{J}| d \eta} \\
& {\left[E^{2}\right]=\int_{\partial \Omega}\left[\boldsymbol{B}^{2}(\eta)\right]^{T}[\boldsymbol{D}]\left[\boldsymbol{B}^{2}(\eta)\right]|\boldsymbol{J}| d \eta}
\end{aligned}
$$


Additionally, $\mathrm{P}$ represents the nodal forces and $u_{h}$ the calculated displacements on the boundary. It can be seen that eqn. 14 must be satisfied on the boundary and eqn. 15 must be satisfied in the domain. Eqn. 15 is termed the scaled boundary finite element equation in displacement.

\subsubsection{Solution procedure}

A general solution of the scaled boundary finite element equation is assumed as a power series of following form

$$
\begin{array}{r}
\{\boldsymbol{u}(\xi)\}=c_{1} \xi^{\lambda_{1}}\left\{\boldsymbol{\phi}_{1}\right\}+c_{2} \xi^{\lambda_{2}}\left\{\boldsymbol{\phi}_{2}\right\}+\ldots+c_{n} \xi^{\lambda_{n}}\left\{\boldsymbol{\phi}_{n}\right\} \\
=[\boldsymbol{\phi}] \xi^{\lceil\boldsymbol{\lambda}\rfloor}\{\boldsymbol{c}\}
\end{array}
$$

where $\lambda_{i}$ and $\left\{\phi_{i}\right\}$ are the corresponding eigenvalues and eigenvectors. The integration constants $c_{i}$ are dependant on the boundary conditions. Visually, this solution may be interpreted as resembling the mode superposition method for standard finite element schemes: The eigenvector $\left\{\phi_{i}\right\}$ can be thought of as a modal displacement vector of the boundary nodes, while $\lambda_{i}$ is the accompanying modal scaling factor in radial $\xi$ direction.

Upon substitution of the general solution into the scaled boundary finite element equation in displacements, the following quadratic eigenvalue equation results:

$$
\begin{array}{r}
{\left[\lceil\boldsymbol{\lambda}]^{2}\left[\boldsymbol{E}^{\mathbf{0}}\right]-\lceil\boldsymbol{\lambda}]\left[\left[\boldsymbol{E}^{\mathbf{1}}\right]^{T}-\left[\boldsymbol{E}^{\mathbf{1}}\right]\right]-\left[\boldsymbol{E}^{\mathbf{2}}\right]\right]\{\boldsymbol{\phi}\}=\left\{\begin{array}{r}
\{\mathbf{0}\} \\
\{\boldsymbol{q}\}=\left[\left[\boldsymbol{E}^{\mathbf{1}}\right]^{T}-\lceil\boldsymbol{\lambda}]\left[\boldsymbol{E}^{\mathbf{0}}\right]\right]\{\boldsymbol{\phi}\}
\end{array}\right.}
\end{array}
$$

Eqn. $18 b$ thus represents a modal interpretation of the boundary forces and consequently can be regarded as nodal force modes required to balance the corresponding displacement modes on the boundary. As a result, all quantities associated with $\phi$ must correlate to displacements by assumption of the general solution, while q directly links to the modal forces on the boundary.

It has been shown that from a fracture mechanics standpoint, the linearisation of the above quadratic equations is beneficial [13]. However, this comes at the price of doubling the amount of equations needed to be solved and re- sults in the following formulation:

$$
[Z]\left[\begin{array}{l}
\phi \\
q
\end{array}\right]=\lceil\lambda\rfloor\left[\begin{array}{l}
\phi \\
q
\end{array}\right]
$$

where

$$
[\boldsymbol{Z}]=\left[\begin{array}{cc}
{\left[\boldsymbol{E}^{\mathbf{0}}\right]^{-1}\left[\boldsymbol{E}^{\mathbf{1}}\right]^{T}} & -\left[\boldsymbol{E}^{\mathbf{0}}\right]^{-1} \\
{\left[\boldsymbol{E}^{\mathbf{1}}\right]\left[\boldsymbol{E}^{\mathbf{0}}\right]^{-1}\left[\boldsymbol{E}^{\mathbf{1}}\right]^{T}-\left[\boldsymbol{E}^{\mathbf{2}}\right]} & -\left[\boldsymbol{E}^{\mathbf{1}}\right]\left[\boldsymbol{E}^{\mathbf{0}}\right]^{-1}
\end{array}\right]
$$

and $[\boldsymbol{Z}]$ is a Hamiltonian matrix, which dictates symmetry about the real and the imaginary axis for all eigenvalues.

Based on the symmetry of the eigenvalues, their contributions towards the solution should be separated accordingly. The same applies analogously to the integration constants $[\boldsymbol{c}]$.

$$
[\boldsymbol{Z}]\left[\begin{array}{l}
\boldsymbol{\phi} \\
\boldsymbol{q}
\end{array}\right]=\lceil\boldsymbol{\lambda}\rfloor\left[\begin{array}{l}
\boldsymbol{\phi} \\
\boldsymbol{q}
\end{array}\right]=\left[\begin{array}{ll}
{\left[\phi_{1}\right]} & {\left[\phi_{2}\right]} \\
{\left[q_{1}\right]} & {\left[q_{2}\right]}
\end{array}\right]\left[\begin{array}{ll}
\left\lceil\lambda^{-}\right\rfloor & \\
& \left\lceil\lambda^{+}\right\rfloor
\end{array}\right]
$$

where the subscripts "+" and "--" denote positive and negative eigenvalues respectively.

This corresponds to splitting the bounded response from the unbounded response.

In order to determine the stiffness matrix of the domain, the eigenvalues and eigenvectors are substituted into the general solution (eqn. 17):

$$
\{\boldsymbol{u}(\xi)\}=\left[\boldsymbol{\phi}_{\mathbf{1}}\right] \xi^{\left\lceil\boldsymbol{\lambda}^{+}\right\rfloor}\left\{\boldsymbol{c}_{\mathbf{1}}\right\}+\left[\boldsymbol{\phi}_{\mathbf{2}}\right] \xi^{\left\lceil\boldsymbol{\lambda}^{-}\right\rfloor}\left\{\boldsymbol{c}_{\mathbf{2}}\right\}
$$

Using the previous observation and the fact that a bounded domain is to be analysed, the second part of eqn. 22 is discarded. Further, in order to determine the stiffness matrix of the bounded domain, the displacements at the boundary $\{\boldsymbol{u}(\xi=1)\}$ are compared to their corresponding equivalent nodal forces $\left\{\boldsymbol{P}_{\text {bounded }}\right\}=\left[\boldsymbol{q}_{\mathbf{1}}\right]\left\{\boldsymbol{c}_{\mathbf{1}}\right\}$, resulting from the integration constants enforcing the boundary conditions on the force modes. This is achieved by first evaluating eqn. 22 at $\xi=1$ and then solving for the integration constants $\left\{\boldsymbol{c}_{\mathbf{1}}\right\}$ :

$$
\left\{\boldsymbol{c}_{\mathbf{1}}\right\}=\left[\Phi_{1}\right]^{-1}\{\boldsymbol{u}(\xi=1)\}
$$

Next, substituting eqn. 23 into the expression for the equivalent nodal forces $\left\{\boldsymbol{P}_{\text {bounded }}\right\}$ results in:

$$
\left\{\boldsymbol{P}_{\text {bounded }}\right\}=\left[\boldsymbol{q}_{\mathbf{1}}\right]\left[\Phi_{1}\right]^{-1}\{\boldsymbol{u}(\xi=1)\}
$$

Consequently, the stiffness matrix of a bounded domain is given as:

$$
K_{\text {bounded }}=+\left[\boldsymbol{q}_{\mathbf{1}}\right]\left[\Phi_{1}\right]^{-1}
$$


The back calculation of strains and stresses follows by first determining the integration constants (eqn. 23), then the general solution (eqn. 22) and finally the stresses (eqn. 11) and strains (eqn. 9) follow.

\subsection{Calculation of stress intensity factors}

One unique feature of SBFEM is the ease with which the stress intensity factors (SIFs) can be calculated. As opposed to other methods, no modification to the solution procedure is necessary and they can be extracted conveniently in post-processing. This stems from the fact that an analytical solution for displacements, and by extension also the strains and stresses, is found in radial direction. By placing the scaling center at the crack tip, an analytical limit as the stresses approach the crack tip can be taken (Fig. 3).

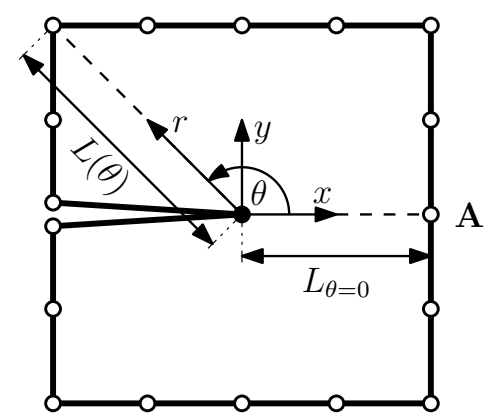

Figure 3: SBFEM domain including a crack and scaling center at crack tip

This results in the following expression for the stresses of one particular element:

$$
\boldsymbol{\sigma}(\xi, \eta)=\sum_{i=1}^{n} c_{i} \xi^{-\lambda_{i}-1} \boldsymbol{\Gamma}_{\boldsymbol{i}}
$$

with $\Gamma_{i}$ representing the corresponding stress modes.

$$
\boldsymbol{\Gamma}_{\boldsymbol{i}}=\left[\begin{array}{l}
\Gamma_{x x} \\
\Gamma_{y y} \\
\Gamma_{x y}
\end{array}\right]_{i}=\boldsymbol{D}\left[-\lambda_{i} \boldsymbol{B}_{\mathbf{1}}(\boldsymbol{\eta})+\boldsymbol{B}_{\mathbf{2}}(\boldsymbol{\eta})\right] \boldsymbol{\Phi}_{\boldsymbol{i}}
$$

It can be shown, that the only modes contributing to a singular response are within the range of $-1<\lambda<0$. Thus, $\boldsymbol{\Phi}_{\boldsymbol{i}}$ represents a submatrix of the displacement modes corresponding to the intersection of a sigular mode column and the associated DOFs rows of the element encompassing node A. By matching the expression for the singular modes with the definition of the SIFs on the boundary and applying the boundary conditions via integration constants, an expression for the mode I and mode II SIFs can be found:

$$
\left[\begin{array}{c}
K_{I} \\
K_{I I}
\end{array}\right]=\sqrt{2 \pi L_{0}}\left[\begin{array}{c}
\sum_{i=I, I I} c_{i} \Gamma_{y y}\left(\eta=\eta_{A}\right)_{i} \\
\sum_{i=I, I I} c_{i} \Gamma_{x y}\left(\eta=\eta_{A}\right)_{i}
\end{array}\right]
$$

Since the calculation of SIFs is based purely on stresses, favourable post-processing techniques are directly applicable. Using a stress recovery technique such as the Super-convergent Patch Recovery (SPR) theory ([29], [30]), the raw stress field may be recovered resulting in significantly improved results. Next to the fact that only the singular modes have to be smoothed on the boundary, numerical experiments have shown that excellent results can still be achieved by only recovering the stresses over a few element in the general crack extension direction, further reducing computational cost. Furthermore, an effective and computationally economical error estimator [31] for SIFs is possible based on the difference between recovery and raw stresses (Fig.10). As opposed to XFEM though, no additional enrichment terms must be included and thus the need for integration of singular terms is eliminated. Since in SBFEM all elements can be treated equally, many issues pertaining to the condition number of the stiffness matrix can be avoided. Additionally, no assumptions on the type of singularity to include in the crack tip enrichment function is necessary. On top of that SBFEM is also exempt of parameter specific dependencies, such as the radius of integration and enrichment, as encountered in XFEM.

\section{Numerical Examples}

In a first numerical example, the convergence characteristics of SBFEM are studied. In a second numerical example, its accuracy and speed of computing SIFs is compared to XFEM and ABAQUS. SBFEM and XFEM are both implemented in Matlab ${ }^{\circledR}$ 2015b. In ABAQUS 6.14-1, the contour integral was used to compute the SIFs. All calculation were performed on an Intel XEON E3-1225 v3. 


\subsection{Square plate with edge crack, mode II}

The square, homogeneous domain with an edge crack, as described in fig. 4 , is considered. For this example, an analytical solution exists [32] for the displacements, stresses and SIFs.

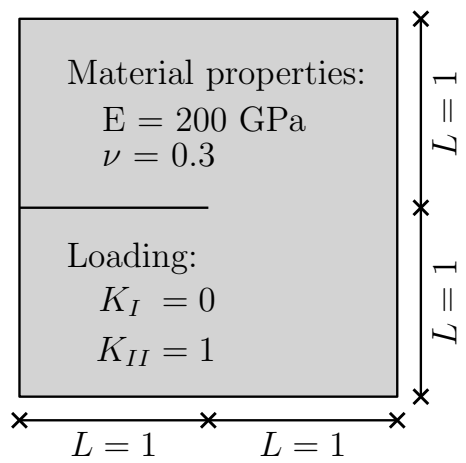

Figure 4: Problem domain with material properties and loading of example 1

In this experiment, a mode II plane stress loading case was considered, resulting from setting $K_{I}=0$ and $K_{I I}=1$, that is setting the mode I SIF to zero and prescribing a mode II SIF equal to one. Based on this choice, the resulting exact displacements were enforced as boundary conditions for each node. Consequently, the $\mathrm{L}_{2}$ norm of displacement error can be easily formulated.

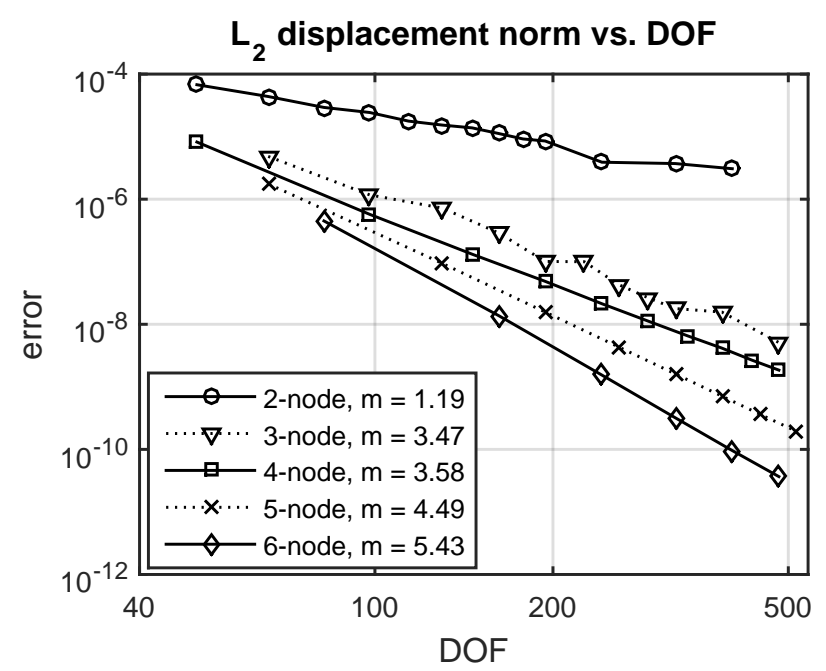

Figure 5: Error in $\mathrm{L}_{2}$ displacement norm vs DOF

Fig. 5 illustrates the convergence behaviour of SBFEM, where the displacement error is plotted against DOF of the domain, including higher order elements. It becomes apparent that linear, 2-node elements perform significantly inferior to the higher order elements. Additionally, there tends to be minor oscillation in the convergence behaviour that subsides as higher order elements are used. This can be attributed to the stability of the routine used to solve the eigen-problem. During the eigen-decompositon of the Hamiltonian matrix Z, a small stabilizing parameter $\epsilon$ is added. Numerical experiments have shown that though the introduction of $\epsilon$ does lead to a correct solution, the convergence is impacted marginally. Consequently, it must be noted, that although SBFEM converges rapidly, only close to optimal convergence is achieved.

Similarly, the $\mathrm{L}_{2}$ norm of stress error can be evaluated as seen in Fig. 6.

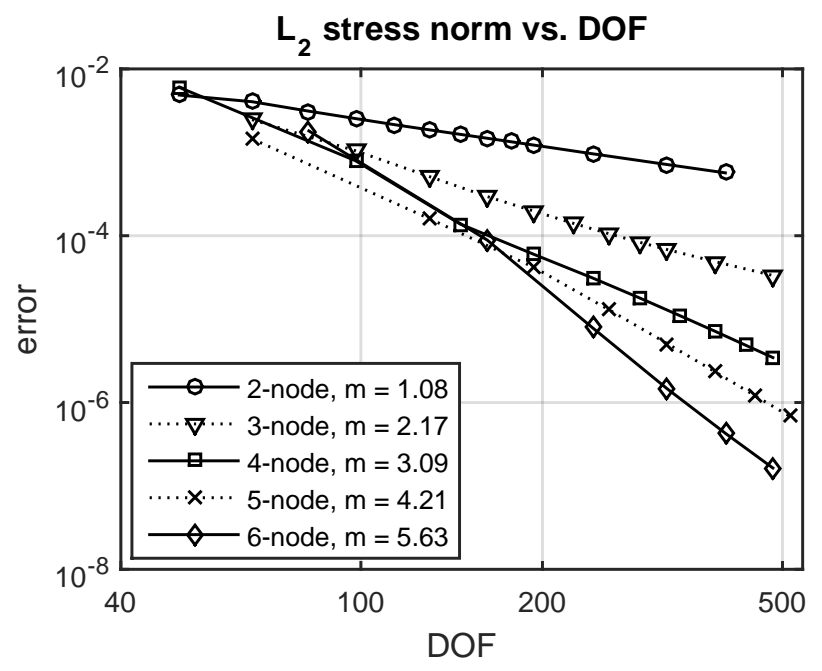

Figure 6: Error in $\mathrm{L}_{2}$ stress norm vs DOF

For this, the stresses were first computed and then smoothed using SPR theory. Finally, the van Mises stresses were computed and used as the basis for the calculation. Remarkably, all convergence lines contain an abrupt change in slope. This can be primarily attributed to the fact that SBFEM performs poorly, if only one element is employed to model one side of a domain as is the case in this example. Because the solution for SBFEM is found as a superposition of modes, having only one element to 
model a part of the boundary significantly limits the mode shapes that can be reflected. Thus, it is recommended to use at least two elements per side when modelling a domain with abrupt changes in element orientation. Removing the computations where only one element is used to model a side surface, excellent convergence properties are obtained.

The rapid convergence properties of SBFEM can also be observed when examining the SIFs. Indeed very few DOF are necessary in order to approximate the exact solution as can be seen in Fig. 7 .

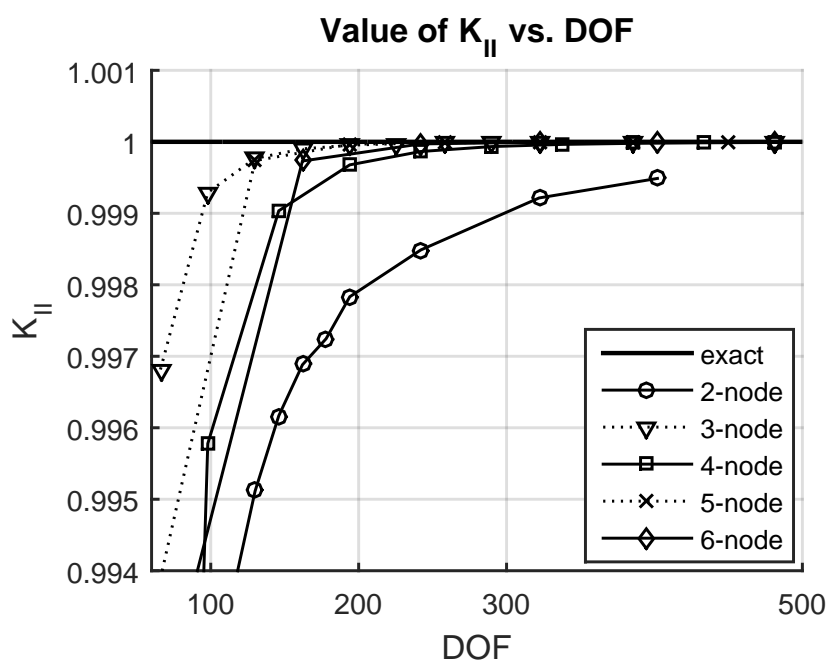

Figure 7: Absolute values of numerically computed $K_{I I}$ vs DOF

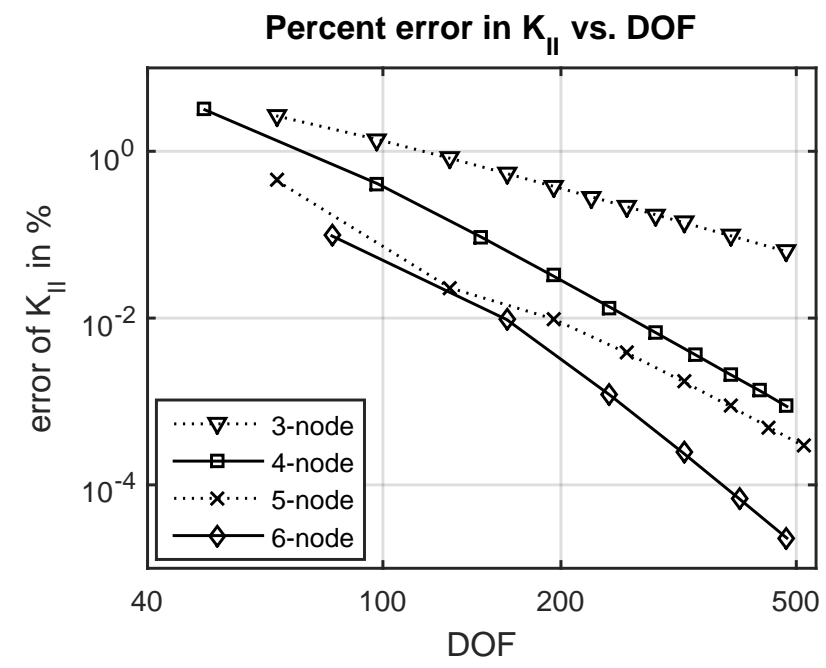

Figure 8: Percent error in numerically computed values for $K_{I I}$ vs DOF
With the aim of facilitating visual inspection, the same response as in Fig. 7 is plotted in Fig. 8. however this time as percent error in SIFs between the numerically computed values and the exact solution. From this second figure, it would seem that the even and odd noded elements converge at different rates. If this hold true for other numerical examples is still to be determined.

Nevertheless, as a main goal of coupling SBFEM with a multiscale approach is to ideally increase the accuracy with which the SIF can be computed while decreasing computational cost, Fig. 9 compares percent error in calculated SIFs to wall clock time. As can be seen, in order to achieve sub percent accuracy in SIFs for this benchmark problem, it is not necessary to compute for longer than 0.1 seconds. As again SPR theory was applied to recover the singular stresses and thus calculate the SIFs, this enables the construction of a straight forward error estimator. Fig. 10 demonstrates the effectivity index for the case of 3-node elements. Naturally, as the numerical solution obtained by SBFEM converges rapidly with only few DOF, the recovered SIFs closely conform to the exact solution, which ultimately contributes to the accuracy of the error estimator and its effectivity index.

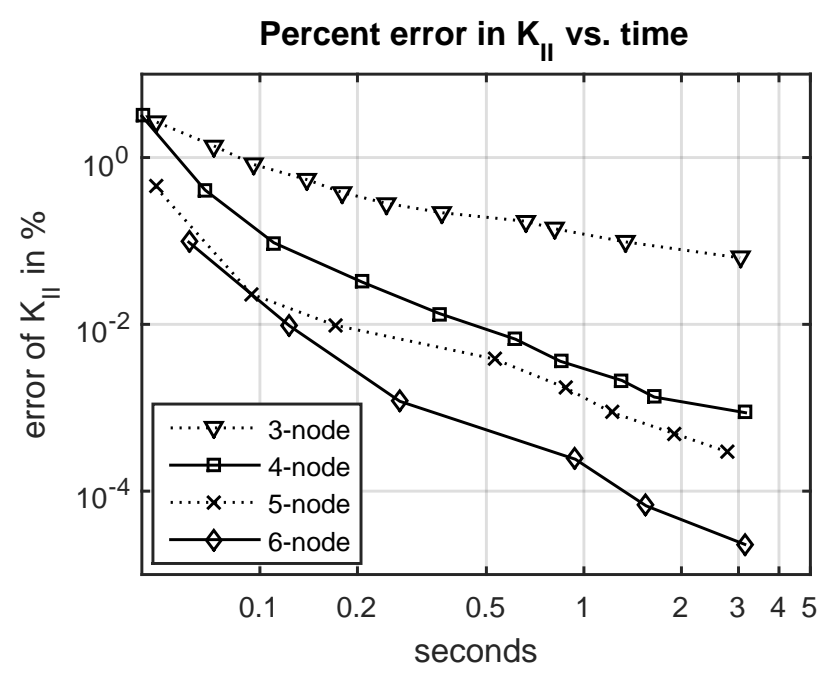

Figure 9: Percent error in numerically computed values for $K_{I I}$ vs computational time 


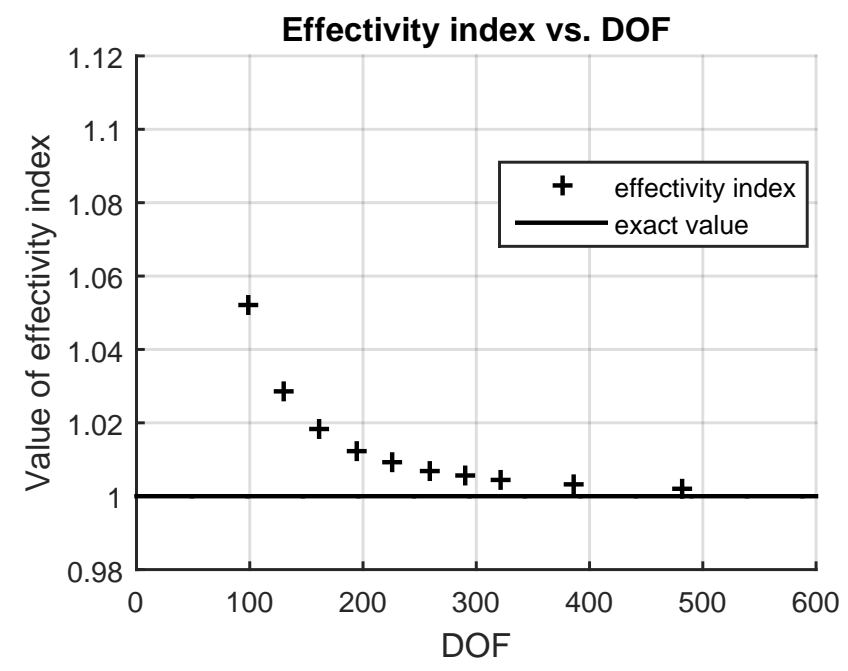

Figure 10: Effectivity index of error estimator

\subsection{Rectangular plate with edge crack}

This next numerical example is a rectangular plate with an edge crack under mode II loading, as is depicted in Fig. 11 .

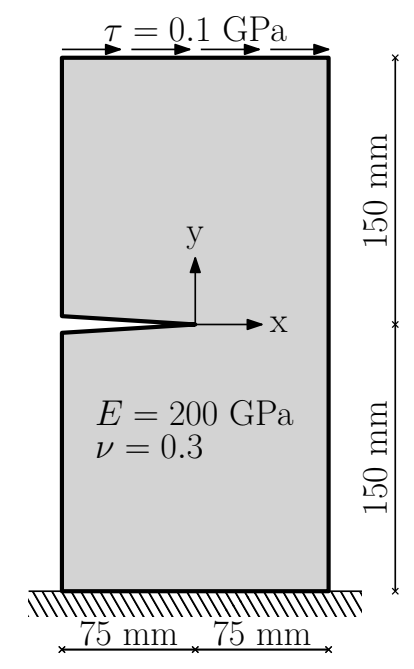

Figure 11: Problem domain, material properties and loading of example 2

Based on the geometry of the domain in conjunction with the boundary conditions, the mode I and mode II SIFs, $K_{I}$ and $K_{I I}$ respectively, will both take on values other then zero. The aim is thus to contrast SBFEM with its nearest competitors, XFEM as well as a software package ABAQUS, all with regards to the computation of SIFs. To this end, the effect of stress smoothing on accuracy will briefly be discussed first. Then the accuracy of the computed
SIFs will be plotted vs computational wall clock time required to obtain the SIFs.

Qualitatively, the stress recovery method based on SPR theory has been previously described as being highly advantageous. However, its effects have previously not been quantified before in the context LEFM. In order to illustrate the effect, the mode I SIF $K_{I}$ is compared to the exact solution. Fig. 12 left, plots the deviation of the numerically computed value, based on raw stresses, to the exact solution as a percentage versus computational time. In Fig. 12 right, on the other hand, the recovered stresses were used to calculate the SIFs. A couple of effects can be observed. First, all solutions based on the recovered stresses converge to the exact solution and without oscillation in accuracy. Thus, a higher amount of DOF is guaranteed to produce a closer estimate of the exact SIFs. Next, all meshes of higher order elements obtain higher accuracy after a stress recovery method is applied. Further, there is no guarantee that the numerically calculated SIFs approach the exact solution strictly from the positive of negative side alone. This is in part due to the modal superposition characteristic of the SBFEM solution. Finally, the lower order the element, the higher the deviation between raw and recovered SIFs.

Recalling that the SBFEM and XFEM implementations are written in Matlab as prototype code while ABAQUS is a commercial software, the comparison of computational time required must always be examined in context. Further, in order to provide a parametric study in XFEM similar to the one performed in SBFEM based on higher order elements, the radius of tip enrichment $\left(r_{d}\right)$ was modified. Calculations were performed with the enrichment radius ranging from 1.55-3.55 times an element length. Figs. 13 and 14 illustrate the SIFs $K_{I}$ and $K_{I I}$ calculated by all three numerical methods. The marginal 'overshooting' of XFEM is attributed to the slight difference in which the boundary conditions were applied in SBFEM and ABAQUS. Nevertheless, it is indisputable that it is possible to compute the SIFs, but 

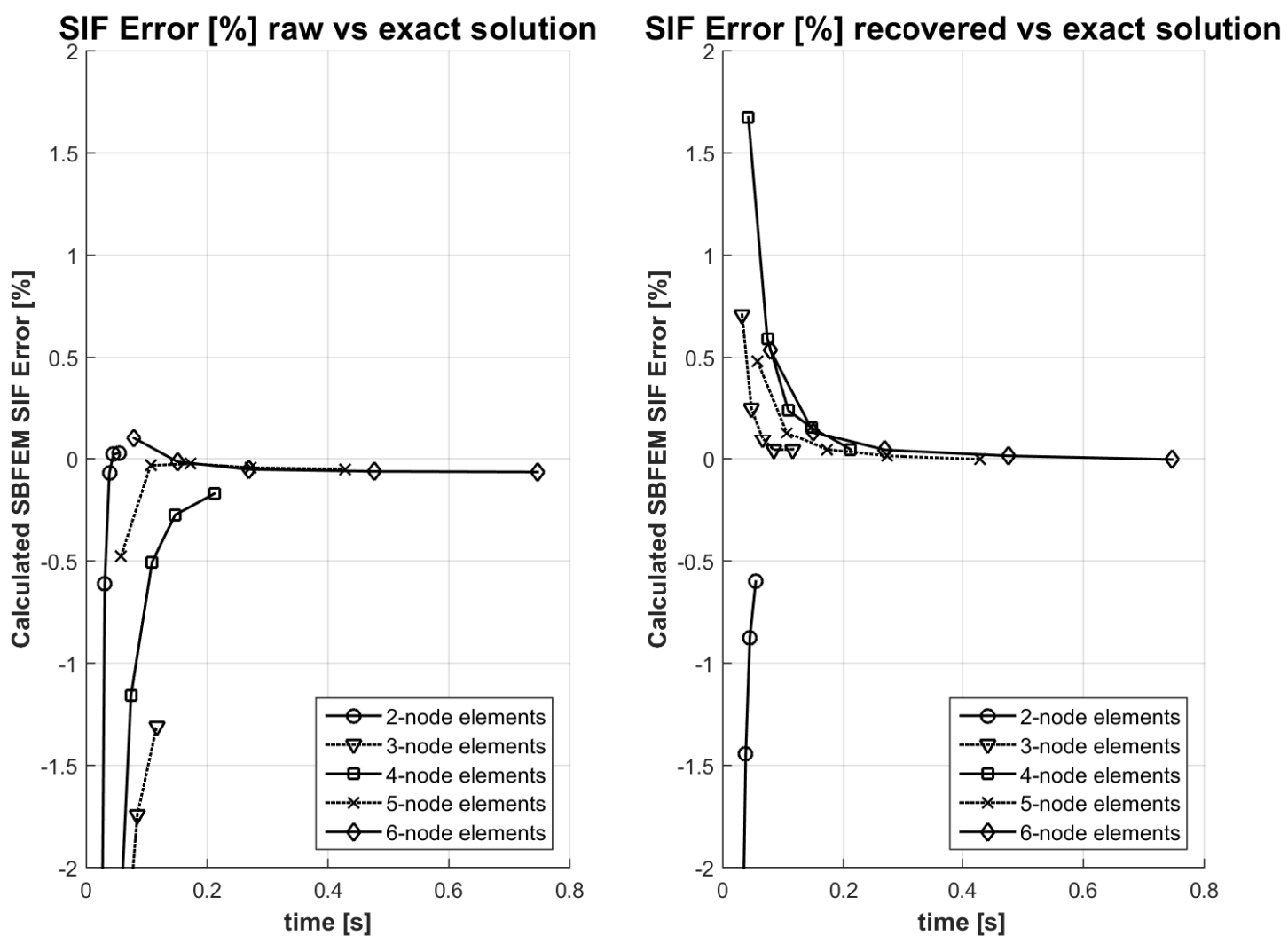

Figure 12: Comparison between SIFs computed using raw (left) and recovered (right) stresses

with the same accuracy one order of magnitude faster when using SBFEM in place of XFEM. Furthermore, the SBFEM approach is slightly more accurate for the same amount of time allocated on computation as the commercial software ABAQUS. It is interesting to note that the SIF $K_{I}$ approaches the exact value from the upper side, while the SIF $K_{I I}$ does so from the lower side in this particular example.

\section{Conclusion}

SBFEM combines many of the benefits from BEM and FEM into one method, while offering unique features of its own, especially with regards to LEFM and the calculation of SIFs. The semi-analytical nature of SBFEM allows to derive formulations for the SIFs based directly on their definitions, without the need to alter the solution process in any form based on a priori knowledge. As in SBFEM the SIF calculation is based purely on stresses, stress recovery techniques such as SPR theory may be applied to gain additional accuracy at negligible computa- tional cost. Furthermore, by contrasting SIFs computed with the raw stresses to those determined by the recovered ones, an effective error estimator can be formulated.

Finally, comparing SBFEM to XFEM and ABAQUS indicates that SBFEM is an order of magnitude faster than XFEM for the examples presented and matches the computational speed of ABAQUS at equal or better accuracy. With this in mind, SBFEM appears to be a promising numerical method to couple with existing multiscale schemes for the purpose of efficiently and accurately including fracture. This is a direction of future research.

\section{Acknowledgements}

This research is supported by the Swiss National Science Foundation (SNSF), under Project \# 200021_153379, A Multiscale Hysteretic XFEM Scheme for the Analysis of Composite Structures. The authors would further like to thank Dr. Sassan Mohasseb, for his constructive input. 


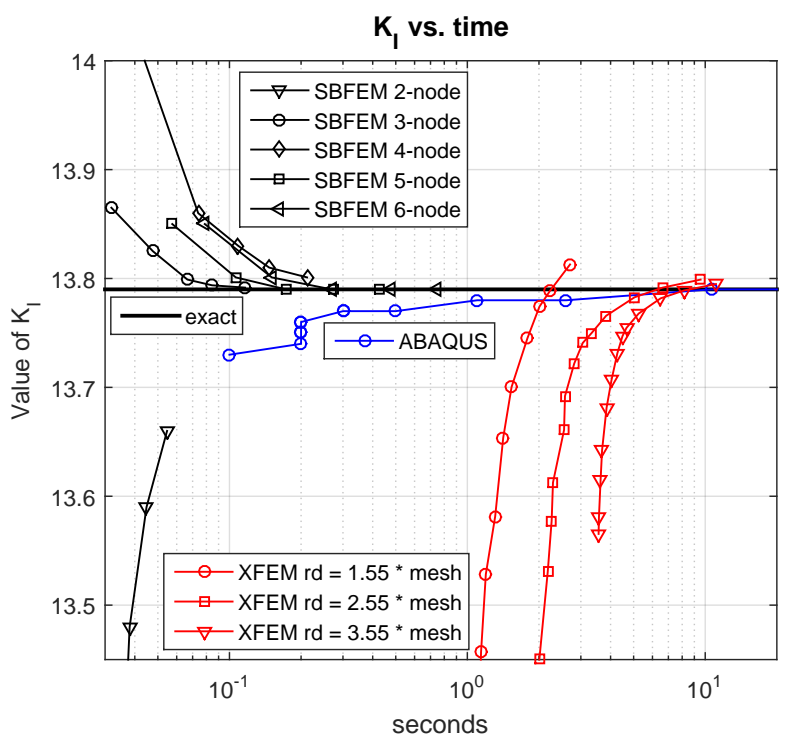

Figure 13: Absolute values of $K_{I}$ vs computational time

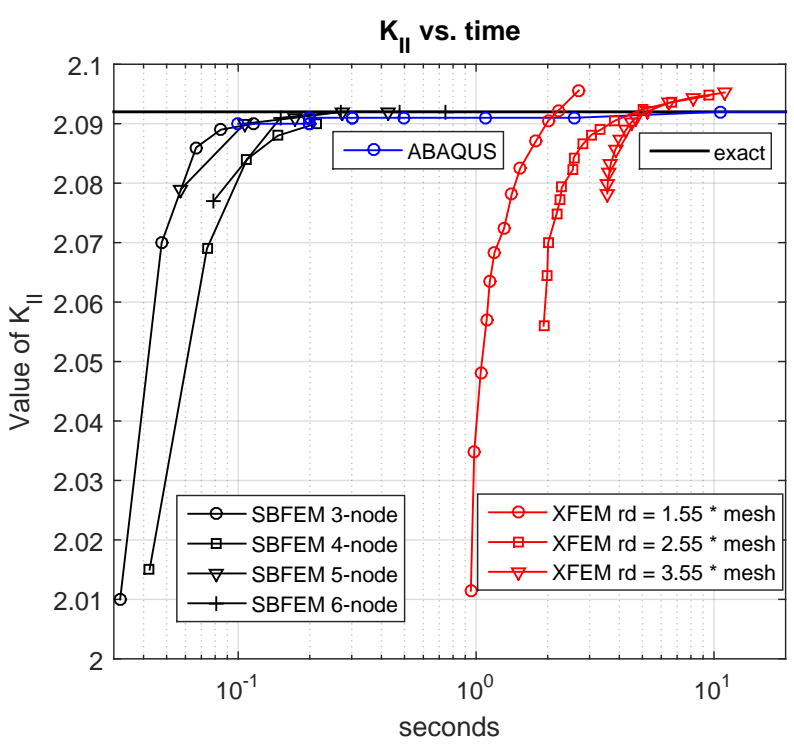

Figure 14: Absolute values of $K_{I I}$ vs computational time

\section{REFERENCES}

[1] Finite elements in fracture mechanics (Springer, New York, 2013)

[2] O.C. Zienkiewicz, R.L. Taylor, J.Z. Zhu, The finite element method: its basis and fundamentals, seventh edition edn. (Elsevier, Butterworth-Heinemann, Amsterdam, 2013)
[3] N. Mos, J. Dolbow, T. Belytschko, International Journal for Numerical Methods in Engineering 46(1), 131 (1999)

[4] N. Sukumar, D. Chopp, N. Mos, T. Belytschko, Computer Methods in Applied Mechanics and Engineering 190(46-47), 6183 (2001)

[5] A. Gravouil, N. Mos, T. Belytschko, International Journal for Numerical Methods in Engineering 53(11), 2569 (2002)

[6] V. Gupta, C. Duarte, I. Babuka, U. Banerjee, Computer Methods in Applied Mechanics and Engineering 266, 23 (2013)

[7] S. Mohammadi, XFEM fracture analysis of composites (John Wiley \& Sons, Ltd, Chichester, West Sussex, United Kingdom, 2012)

[8] G. Dasgupta, Journal of Applied Mechanics 49(1), 136 (1982)

[9] J.P. Wolf, C. Song, Computer Methods in Applied Mechanics and Engineering 123(1-4), 355 (1995)

[10] J.P. Wolf, C. Song, Finite-element modelling of unbounded media (Wiley, Chichester, England ; New York, 1996)

[11] J.P. Wolf, The scaled boundary finite element method (J. Wiley, Chichester, West Sussex, England ; Hoboken, NJ, USA, 2003)

[12] A.J. Deeks, J.P. Wolf, Computational Mechanics 28(6), 489 (2002)

[13] C. Song, Computer Methods in Applied Mechanics and Engineering 193(23-26), 2325 (2004)

[14] Z. Yang, Engineering Fracture Mechanics 73(12), 1711 (2006)

[15] E.T. Ooi, C. Song, F. Tin-Loi, Z. Yang, International Journal for Numerical Methods in Engineering 91(3), 319 (2012) 
[16] E. Ooi, M. Shi, C. Song, F. Tin-Loi, Z. Yang, Engineering Fracture Mechanics 106, 1 (2013)

[17] C. Song, International Journal for Numerical Methods in Engineering 77(8), 1139 (2009)

[18] Z.J. Yang, A.J. Deeks, H. Hao, International Journal for Numerical Methods in Engineering 70(12), 1387 (2007)

[19] H. Man, C. Song, T. Xiang, W. Gao, F. Tin-Loi, International Journal for $\mathrm{Nu}-$ merical Methods in Engineering 95(4), 331 (2013)

[20] E.T. Ooi, C. Song, F. Tin-Loi, Computer Methods in Applied Mechanics and Engineering 268, 905 (2014)

[21] R. Behnke, M. Mundil, C. Birk, M. Kaliske, International Journal for Numerical Methods in Engineering 99(13), 966 (2014)

[22] Z. Lin, S. Liao, Communications in Nonlinear Science and Numerical Simulation 16(1), 63 (2011)

[23] A.J. Deeks, C.E. Augarde, Computational Mechanics 36(3), 159 (2005)

[24] G. Bird, J. Trevelyan, C. Augarde, Engineering Analysis with Boundary Elements 34(6), 599 (2010)
[25] M.C. Genes, S. Kocak, International Journal for Numerical Methods in Engineering 62(6), 798 (2005)

[26] S. Natarajan, C. Song, International Journal for Numerical Methods in Engineering 96(13), 813 (2013)

[27] G. Lin, Y. Zhang, Z. Hu, H. Zhong, Science China Physics, Mechanics and Astronomy 57(2), 286 (2014)

[28] N. Khaji, M. Khodakarami, Engineering Analysis with Boundary Elements 35(6), 845 (2011)

[29] O.C. Zienkiewicz, J.Z. Zhu, International Journal for Numerical Methods in Engineering 33(7), 1331 (1992)

[30] O.C. Zienkiewicz, J.Z. Zhu, International Journal for Numerical Methods in Engineering 33(7), 1365 (1992)

[31] A.J. Deeks, J.P. Wolf, International Journal for Numerical Methods in Engineering 54(4), 557 (2002)

[32] H.L. Ewalds, R.J.H. Wanhill, Fracture mechanics (E. Arnold ; Delftse Uitgevers Maatschappij, London : Delft, Netherlands, 1984) 